\title{
Prediction Model of Wind Speed and Direction using Deep Neural Network
}

\author{
Anggraini Puspita Sari a,b,1,*, Hiroshi Suzuki ${ }^{\mathrm{a}, 2}$, Takahiro Kitajima ${ }^{\mathrm{a}, 3}$, Takashi Yasuno ${ }^{\mathrm{a}, 4}$, Dwi \\ Arman Prasetya ${ }^{b, 5}$ \\ ${ }^{a}$ Tokushima University, 2-1 Minamijosanjima-cho, Tokushima, 770-8506, Japan \\ ${ }^{\mathrm{b}}$ University of Merdeka Malang, Jalan Terusan Dieng No. 62-64 Kota Malang, Jawa Timur 65146, Indonesia \\ ${ }^{1}$ anggi@ee.tokushima-u.ac.jp*; ${ }^{2}$ suzuki.hiroshi@tokushima-u.ac.jp; ${ }^{3}$ kitaji-t@ee.tokushima-u.ac.jp; \\ ${ }^{4}$ yasuno.takashi@tokushima-u.ac.jp; ${ }^{5}$ arman.prasetya@unmer.ac.id \\ * corresponding author
}

\begin{tabular}{|c|c|}
\hline & ABSTRACT \\
\hline Keywords & This paper presents the prediction system of wind speed and direction using a feed- \\
\hline Feed-forward & forward backpropagation neural network (FFBPNN). The input of the prediction \\
\hline Backpropagation & system is wind speed and direction which are numerical data and provided by \\
\hline Neural network & Automated Meteorological Data Acquisition System (AMeDAS) in Japan. The \\
\hline Wind speed & performances of the proposed system is evaluated based on mean square error (MSE) \\
\hline Wind direction & $\begin{array}{l}\text { between predicted and observed data. In this paper, we substantiate the usefulness of } \\
\text { the proposed prediction system improving prediction accuracy compared to four } \\
\text { prediction models. }\end{array}$ \\
\hline
\end{tabular}

\section{Introduction}

At present, many countries especially developed countries conduct research about renewable energy which expected to replace fossil fuels in the future and reduce greenhouse effect or global warming due to the emission of carbon dioxide $(\mathrm{CO} 2)$ and methane $(\mathrm{CH} 4)[1]$. Wind power generation is one of the renewable energy and has rapidly attracted attention as one of electric power generation. The output fluctuation due to wind speed and direction is the main matter of wind power generation. Because of this problem and ensure a stable electric supply, the electrical companies regulated supply power using another source power generation.

Therefore, If we can predict the output of wind power generation, we can stabilize the power system effectively and offer this information to the power company which is useful to the stabilize electric power system and devise thermal power plant output[2]. The approach of wind speed prediction mainly divided two categories which numerical and statistical prediction system. The numerical prediction model used numerical weather prediction and needs long processing times so it needs the high performance of the computer. The statistical prediction system uses previous and current observed data to predict future data[3]. Therefore, we propose the statistical prediction system using deep neural network (DNN) especially utilize a feed-forward backpropagation neural network (FFBPNN). The FFBPNN uses to realize more accurate prediction data such as for remain useful life prediction of Francis Turbines[4]. The usefulness proposes the prediction system to improve prediction accuracy and discuss the effect of the prediction system by comparing four prediction model with different optimizers. 


\section{The Proposed Method}

\subsection{Definition and prediction period of wind speed and direction}

The input of the prediction system using wind speed and direction obtained from AMeDAS, Japan at 10 min interval and the point are used in Tokushima city, Tokushima prefecture, Japan. The wind direction expresses 16 direction with $\mathrm{W}$ is west, $\mathrm{E}$ is east, $\mathrm{N}$ is north and $\mathrm{S}$ is south as shown in Fig 1. Firstly, The wind speed and direction can be used input at the prediction system and be changed to $v_{x}(t)$ and $v_{y}(t)$ utilizing the mathematical model as shown in Eqs. (1) and (2),

$$
\begin{aligned}
& v_{x}(t)=v(t) \cdot \cos \theta(t) \\
& v_{y}(t)=v(t) \cdot \sin \theta(t)
\end{aligned}
$$

where $v(t)$ is wind speed $[\mathrm{m} / \mathrm{s}], \theta(t)$ is wind direction $\left[{ }^{\circ}\right], v_{x}(t)$ and $v_{y}(t)$ are $x$ and $y$ components of $v(t)[\mathrm{m} / \mathrm{s}]$.

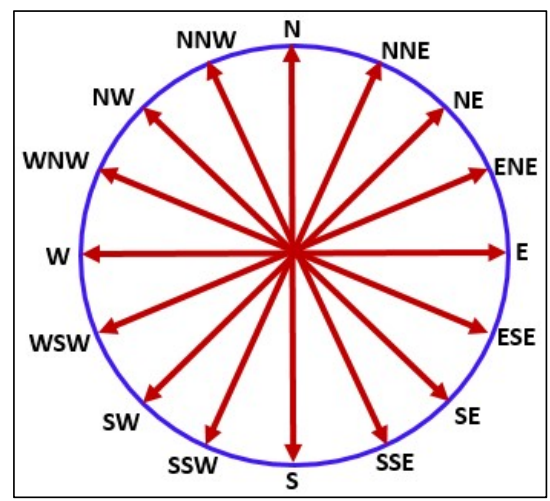

Fig. 1. Definition of Wind Direction

The direction of west-east represents x-axis and north-south represents y-axis as shown in Fig. 2. The prediction period is set to one hour later by considering the capability to adapt thermal power plant and pumped-storage hydroelectricity[5].

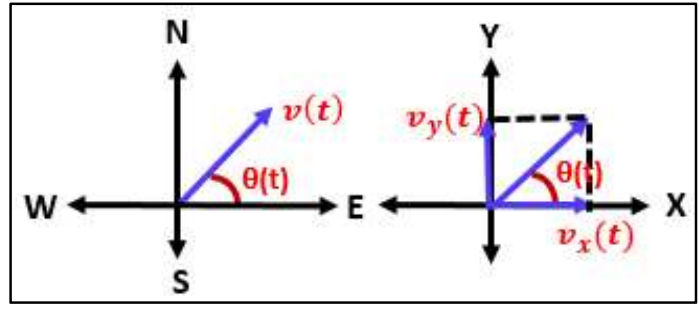

(a)

(b)

Fig. 2. Wind Speed and Direction on Coordinate System

(a) Vector Diagram (b) Definition of Component $v(t)$

\subsection{Network Configuration of Prediction System}

The network configuration of FFBPNN as shown in Fig. 3. The prediction system uses feedforward and backpropagation process to get the prediction result of wind speed and direction. The merit of this process are accuracy improvement and reliability[4]. This prediction system consists of eight layers: one input layer, six hidden layers and one output layer. Every one layer to one layer has weights and biases. The input layer utilizes twenty four data consists twelve data of $v_{x}(t)$ and 
twelve data of $v_{y}(t)$ which time interval $(t)$ is set to 10 min and proposed prediction systems are conducted every two hours.

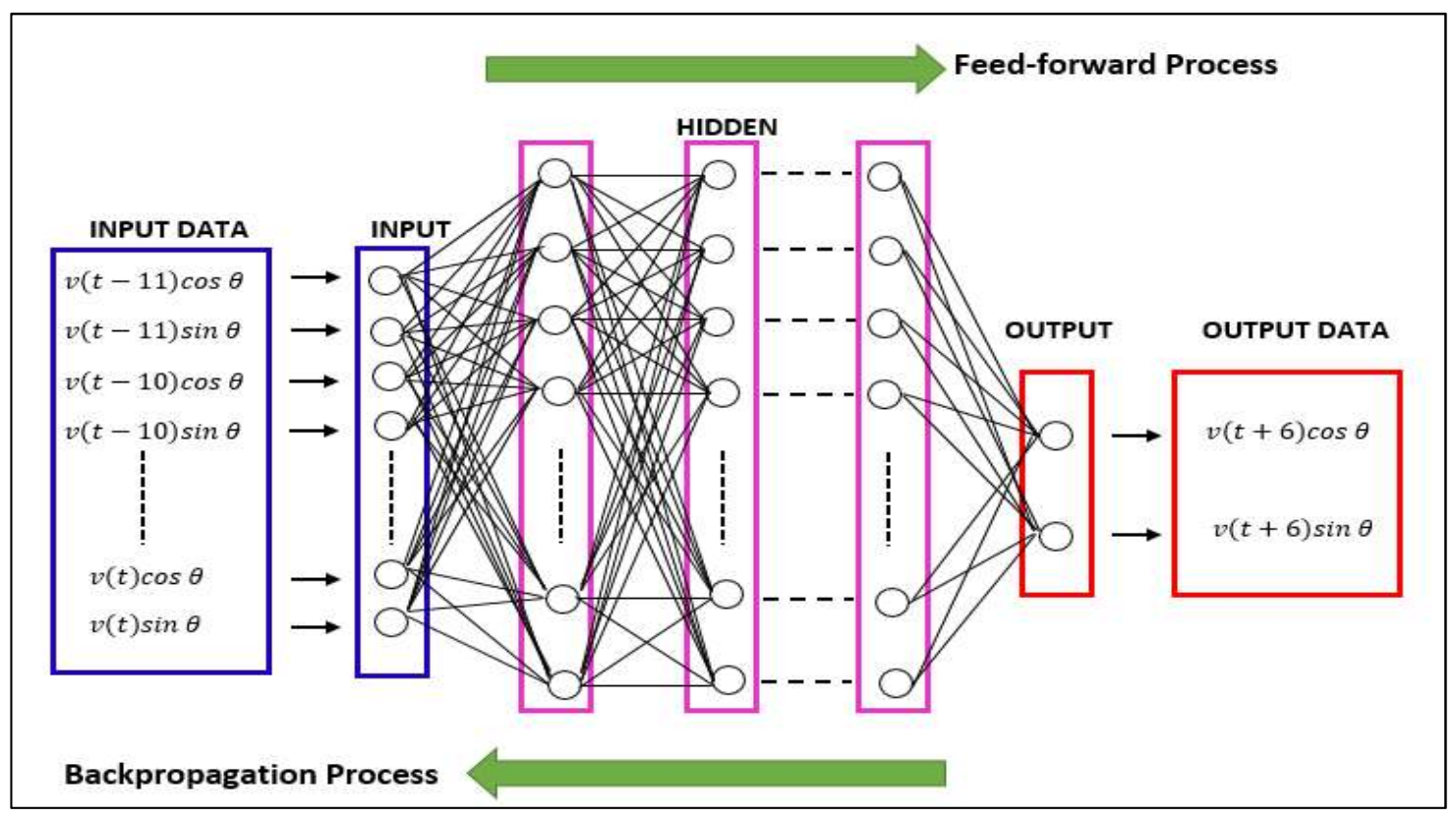

Fig. 3. Network Configuration of FFBPNN

Each hidden layer has 128 neurons. The output data utilizes two data which one data of $v_{x}(t)$ and one data of $v_{y}(t)$ and the result of proposed system is one hour later. The feed-forward process is calculating the predicted output of $v_{x}(t)$ and $v_{y}(t)$ and also the backpropagation process is updating weights and biases. The calculation of input into output neural network as shown in Eqs. (3), (4) and (5). The Eq. (4) is input to hidden layer, Eq. (4) is hidden to hidden layer and Eq. (5) is hidden to output layer[6][7],

$$
\begin{aligned}
& \bar{h}_{1}=\phi\left(\sum_{1}^{l}\left(w_{1}^{l} \bar{x}\right)+b_{1}\right) \\
& \bar{h}_{p+1}=\phi\left(\sum_{p+1}^{l}\left(w_{p+1}^{l} \bar{h}_{p}\right)+b_{p+1}\right) \\
& \bar{o}=\phi\left(\sum_{k+1}^{l}\left(w_{k+1}^{l} \bar{h}_{k}\right)+b_{k+1}\right)
\end{aligned}
$$

where $\bar{x}, \bar{h}, \bar{o}$ are output signal of input layer, hidden layer and output layer, respectively; $b$ is bias; $w$ is weight; $\phi$ is activation function; $p$ and $k$ are number of hidden layer and $l$ is number of neuron.

The prediction system using four models: prediction model 1 using optimizer of adam, prediction model 2 using optimizer of stochastic gradient descent (SGD), prediction model 3 using optimizer of adadelta and prediction model 4 using optimizer of adagrad. These prediction models and persistent model are compared with each other to investigate the results of prediction system.

\section{Method}

\subsection{Dataset}

Table 1 shows the dataset of training, validation and test data. The prediction system using one year data of 2016 for training, one year data of 2017 for validation and one year data of 2018 for test. 
Table 1. MSE of Training and Validation Data

\begin{tabular}{ccc}
\hline Dataset & Period & Total Data \\
\hline Training & 2016 & 52704 \\
\hline Validation & 2017 & 52560 \\
\hline Test & 2018 & 52560 \\
\hline
\end{tabular}

\subsection{Learning Procedure}

The prediction system is executed by python and framework tensorflow. The Parameters for all models as shown in Table 2. The activation function are set the prediction system uses rectified linear units (ReLU). The performances of these prediction systems are evaluated by mean square error (MSE) as shown in Eq. (6),

$M S E=\frac{1}{N} \sum_{i=1}^{N}\left(o_{i}-\hat{o}_{i}\right)^{2}$

where $o_{i}$ is observed value and $\hat{o}_{i}$ is predicted value and $N$ is the number of sample data.

Table 2. Parameter Of All Model

\begin{tabular}{cc}
\hline Description & Data \\
\hline Activation function & ReLU \\
\hline Epoch & 500 \\
\hline Loss function & MSE \\
\hline Neuron of hidden layer & 128 \\
\hline Learning rate & 0.001 \\
\hline Batch size & 100 \\
\hline
\end{tabular}

\section{Results and Discussion}

\subsection{Prediction Result of DNN}

Predicted values of wind speed $(\hat{v}(t))$ and direction $(\hat{\theta}(t))$ are calculated by Eqs. (6) and (7),

$\hat{v}(t)=\sqrt{\left[\hat{v}_{x}(t)\right]^{2}+\left[\hat{v}_{y}(t)\right]^{2}}$

$\hat{\theta}(t)=\tan ^{-1}\left(\frac{\hat{v}_{y}(t)}{\hat{v}_{x}(t)}\right)$

where $\hat{v}_{x}(t)$ and $\hat{v}_{y}(t)$ are $\mathrm{x}$ and y components of predicted $\hat{v}(t)[\mathrm{m} / \mathrm{s}]$.

In order to confirm the performance of each prediction model, we predict wind speed and direction one hour later for each month in 2018. MSE of training data uses 500 epochs for each model as shown in Fig. 4. Table 3 shows MSE of training and validation data. From Fig 4 and Table 3, MSE of training data for model 4 is lower than other models but higher than other models for MSE of validation data. 


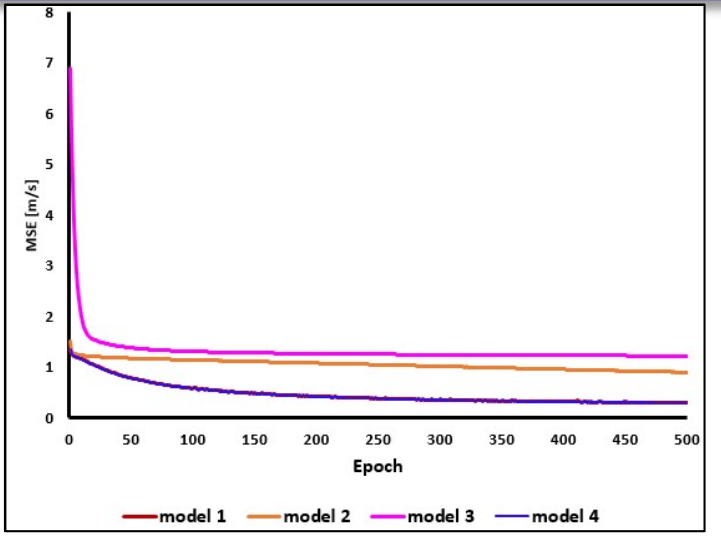

Fig. 4.MSE of Training Data for Each Prediction Model

Table 3. MSE of Training and Validation Data

\begin{tabular}{ccc}
\hline Description & Training $[\mathbf{m} / \mathbf{s}]$ & Validation $[\mathbf{m} / \mathbf{s}]$ \\
\hline Model 1 & 0.4725 & 1.7139 \\
\hline Model 2 & 1.0532 & 1.2883 \\
\hline Model 3 & 1.3282 & 1.3259 \\
\hline Model 4 & 0.4670 & 1.7171 \\
\hline
\end{tabular}

Fig. 5(a) and (b) show comparison between observed and predicted wind speed for each prediction model using test data. From Fig. 5(a) and (b), the predicted results of the model 3 approximate observed data more than other models. The predicted result of $v_{x}(t)$ and $v_{y}(t)$ are evaluated by MSE. The MSE $\hat{v}_{x}(t)$ and $\hat{v}_{y}(t)$ for each model is listed in Table 4. From Table 4, the MSE of model 3 is lower than other models.

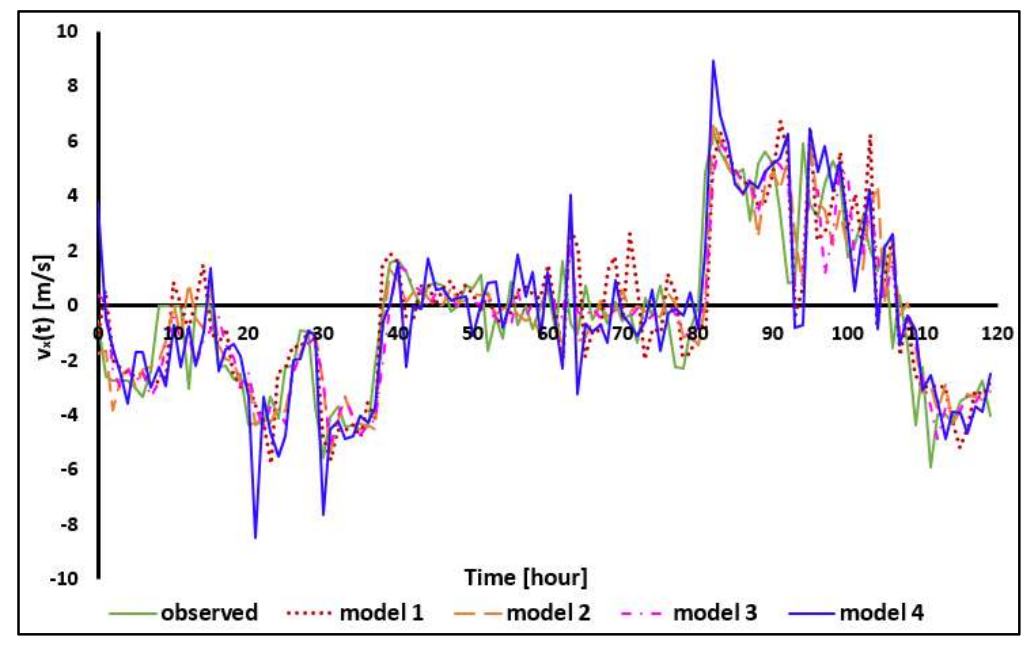

(a) $\hat{v}_{x}(t)$ 


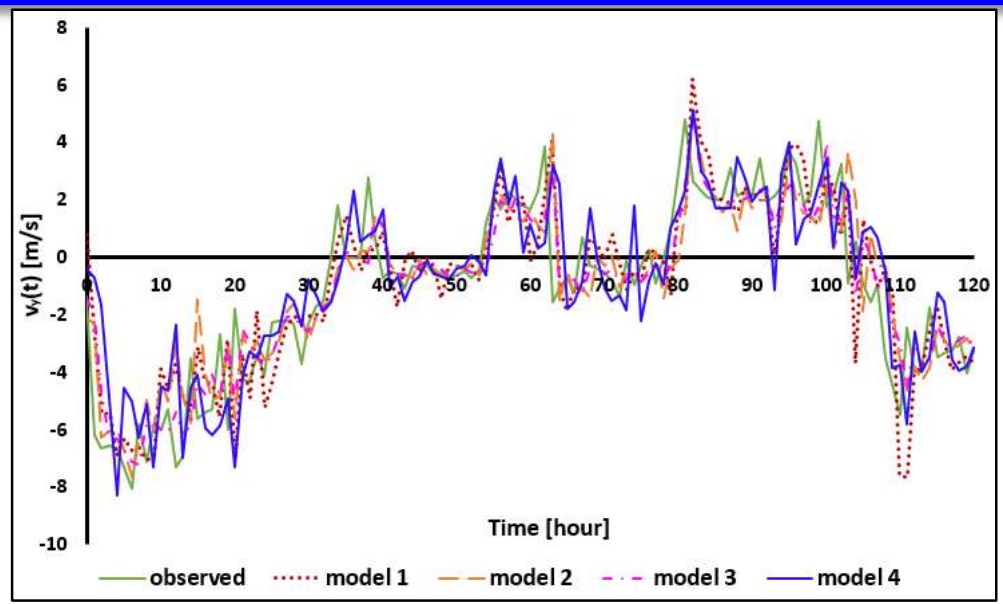

(b) $\hat{v}_{y}(t)$

Fig. 5. (a) and (b) Prediction Results of $v_{x}(t)$ and $v_{y}(t)$ (Mar, 1-5, 2018)

Table 4. MSE of $\hat{v}_{x}(t)$ and $\hat{v}_{y}(t)$ in Test Data

\begin{tabular}{ccc}
\hline Description & $\widehat{\boldsymbol{v}}_{\boldsymbol{x}}(\boldsymbol{t})[\mathbf{m} / \mathbf{s}]$ & $\widehat{\boldsymbol{v}}_{\boldsymbol{y}}(\boldsymbol{t})[\mathbf{m} / \mathbf{s}]$ \\
\hline Model 1 & 2.0592 & 1.8770 \\
\hline Model 2 & 1.5644 & 1.4524 \\
\hline Model 3 & 1.3739 & 1.3163 \\
\hline Model 3 & 2.1665 & 1.9419
\end{tabular}

\subsection{Prediction Results of Wind Speed and Direction}

Fig. 6 shows the observed data compared to the predicted results of $v(t)$ and $\theta(t)$ for the period (Mar, 1-5, 2018) to know the difference between each model. The predicted results of wind speed and direction are evaluated by MSE. The MSE of wind speed and direction for each month as shown in Fig. 7. The MSE of $\hat{v}(t)$ and $\hat{\theta}(t)$ for each model and persistent model are summarized in Table 5. From Fig. 7 and Table 5, we can confirm the prediction accuracy of $v(t)$ and $\theta(t)$ for each model every month (2018). Table 6 shows the improvement rate of each model compared to the persistent model. From Table 6, the prediction system using model 3 is the most effective to more improved prediction accuracy than using other models.

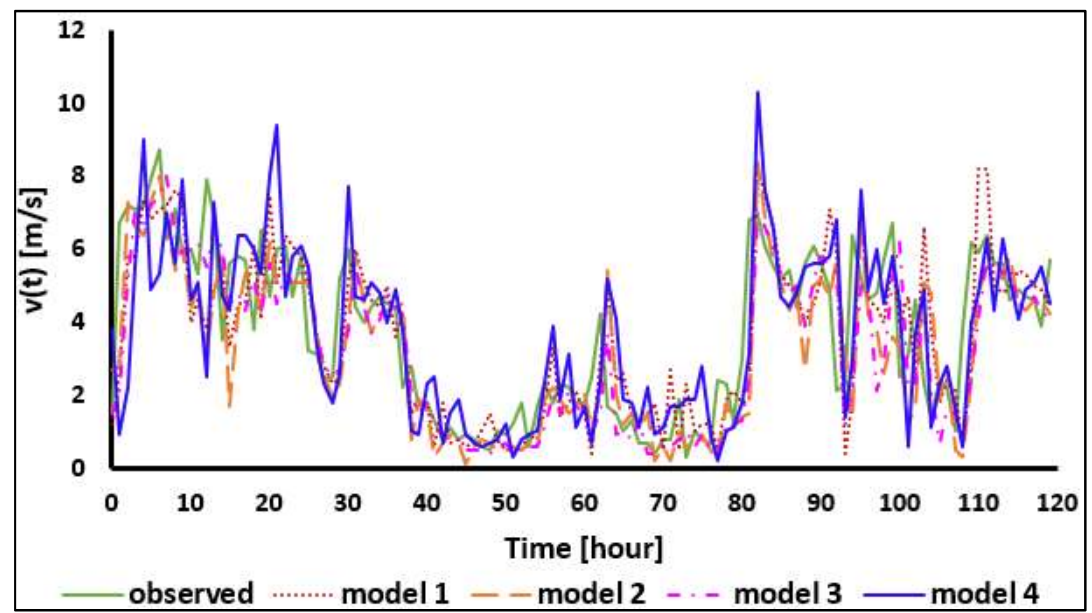

(a) Wind Speed 


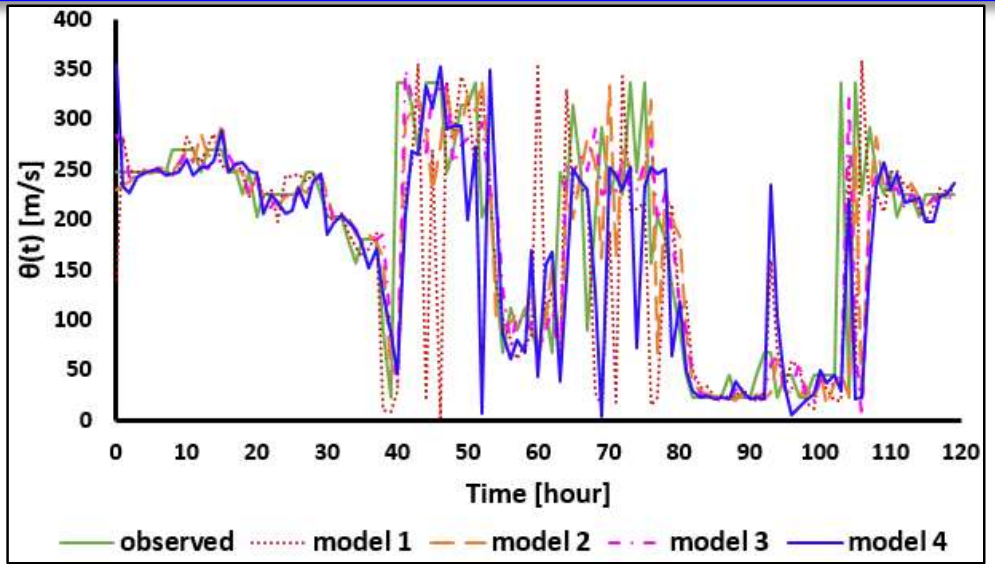

(b) Wind Direction

Fig. 6. Prediction Results of $v(t)$ and $\theta(t)$ (Mar, 1-5, 2018)

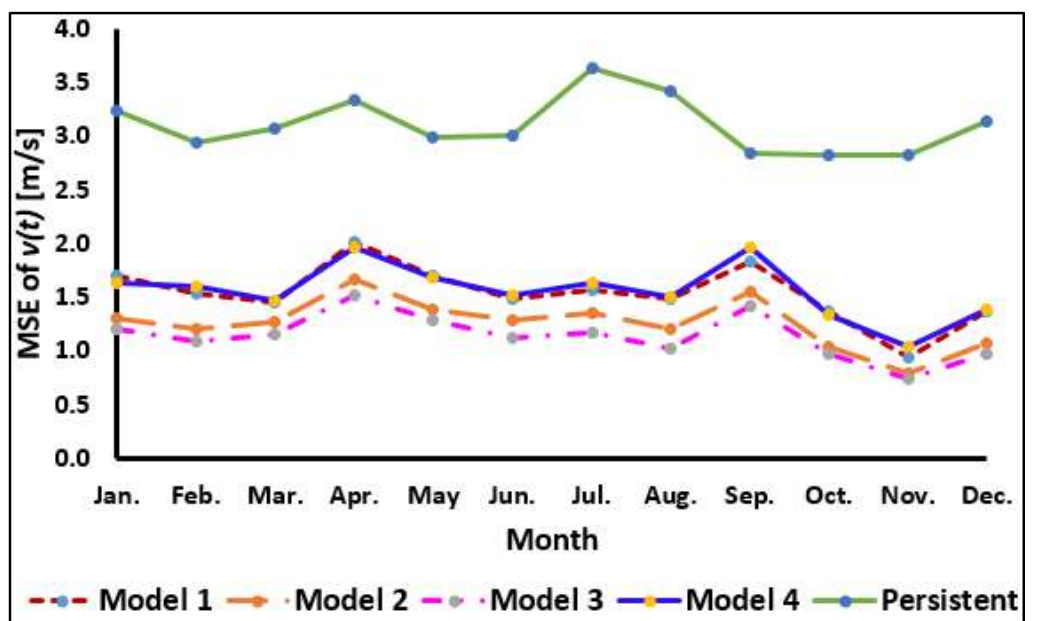

(a) $\hat{v}(t)$

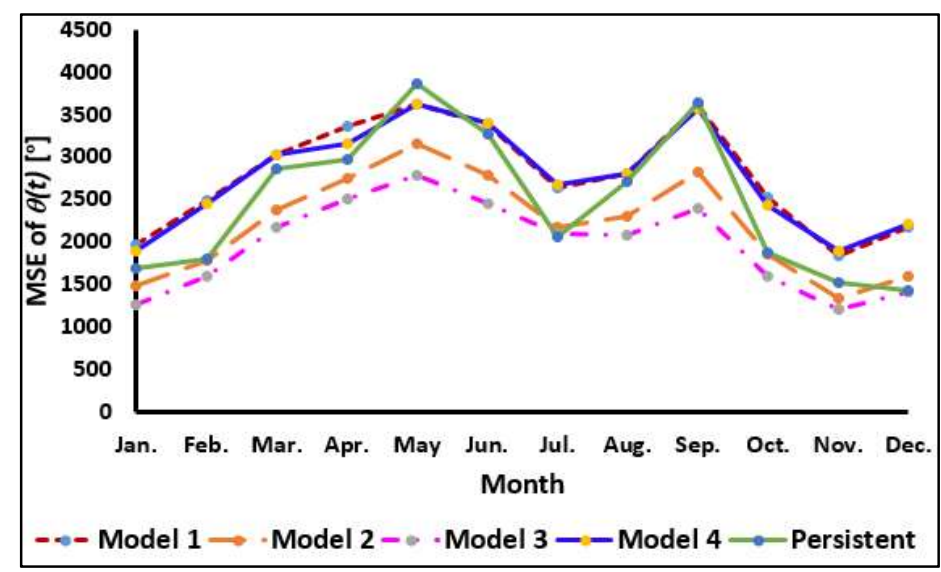

(b) $\hat{\theta}(t)$

Fig. 7. MSE of $\hat{v}(t)$ and $\hat{\theta}(t)$ for Each Month (2018) 
Table 5. $\quad$ MSE OF $\hat{v}(t)$ AND $\hat{\theta}(t)$

\begin{tabular}{ccc}
\hline Description & $\widehat{\boldsymbol{v}}(\boldsymbol{t})[\mathbf{m} / \mathbf{s}]$ & $\widehat{\boldsymbol{\theta}}(\boldsymbol{t})\left[^{\circ}\right]$ \\
\hline Persistent & 3.1081 & 2478.5993 \\
\hline Model 1 & 1.5400 & 2787.2774 \\
\hline Model 2 & 1.2619 & 2205.5557 \\
\hline Model 3 & 1.1396 & 1966.0914 \\
\hline Model 3 & 1.5598 & 2763.6371 \\
\hline
\end{tabular}

Table 6.

IMPROVEMENT RATE FOR EACH MODEL

\begin{tabular}{lllll}
\hline \multirow{2}{*}{ Description } & \multicolumn{4}{c}{ Improvement rate [\%] } \\
\cline { 2 - 5 } & \multicolumn{1}{c}{ Model 1 } & \multicolumn{1}{c}{ Model 2 } & Model 3 & Model 4 \\
\hline$\widehat{\boldsymbol{v}}(\boldsymbol{t})[\mathrm{m} / \mathrm{s}]$ & 50.45 & 59.4 & 63.33 & 49.81 \\
\hline$\widehat{\boldsymbol{\theta}}(\boldsymbol{t})\left[^{\circ}\right]$ & -12.45 & 11.02 & 20.68 & -11.5 \\
\hline
\end{tabular}

\section{Conclusion}

This paper proposed the wind speed and direction prediction model one hour later using deep neural network (DNN). The effectiveness of the prediction model was confirmed from the comparison of the results of the predicted (four prediction model) and observed data. In comparison persistent model, the prediction accuracy of wind speed was improved for the all models, drastically. However, the prediction accuracy of wind direction was improved just model 2 and 3.

The future task will focus on the improvement of the prediction accuracy by searching optimal values of hyperparameters. For example, the prediction model uses the optimum parameters such as number of layer and neurons, and also prediction period for a long time. 


\section{References}

[1] N. L. Panwar, S. C. Kaushik, and S. Kothari, "Role of renewable energy sources in environmental protection: A review," Renew. Sustain. Energy Rev., vol. 15, no. 3, pp. 1513-1524, 2011.

[2] Hitoshi Sori and Takashi Yasuno, "Several Hours Ahead Wind Speed Prediction System Using Correction Recurrent Neural Network," Proc. International Workshop on Nonlinear Circuits, Communications and Signal Processing (NSCP '09), 2009, pp. 621-624.

[3] Y. Miyabe, T. Kitajima, and T. Yasuno, "Wind Speed Prediction Model Using Neural Networks Classified By Observed Wind Speed," Proc RISP International Workshop on Nonlinear Circuits, Communications and Signal Processing (NSCP 2015), 2015, pp. 230-233.

[4] A. Aguinaga, X. Luo, V. Hidalgo, E. Cando, and F. Llulluna, "A feed-forward backpropagation neural network method for remaining useful life prediction of Francis turbines," Proc. World Congr. Mech. Chem. Mater. Eng., no. 126, 2017.

[5] A.P. Sari, H. Suzuki, R. Fukuoka, T. Kitajima, and T.Yasuno "Prediction Model of Wind Speed and Direction using Convolutional Neural Network," Proc. SICE Shikoku Conference, 2019, vol. 2, pp. 2326.

[6] R. E. Neapolitan and R. E. Neapolitan, Neural Networks and Deep Learning, Yorktown Heights, NY: Springer, 2018, pp. 19-47.

[7] S. Samarasinghe, "Neural networks for applied sciences and engineering: from fundamentals to complex pattern recognition," United States: Auerbach, 2007, pp. 73-109. 


\section{This page intentionally left blank}

